\title{
Nouveaux cahiers de la recherche en éducation
}

Durrive, L. (2015). L'expérience des normes. Comprendre

l'activité humaine avec la démarche ergologique. Toulouse : Octares éditions

\section{Samira Mahlaoui}

Volume 19, numéro 2, 2016

URI : https://id.erudit.org/iderudit/1042855ar

DOI : https://doi.org/10.7202/1042855ar

Aller au sommaire du numéro

Éditeur(s)

Faculté d'éducation, Université de Sherbrooke

ISSN

1911-8805 (numérique)

Découvrir la revue

Citer ce compte rendu

Mahlaoui, S. (2016). Compte rendu de [Durrive, L. (2015). L'expérience des normes. Comprendre l'activité humaine avec la démarche ergologique.

Toulouse : Octares éditions]. Nouveaux cahiers de la recherche en éducation, 19(2), 164-168. https://doi.org/10.7202/1042855ar d'utilisation que vous pouvez consulter en ligne.

https://apropos.erudit.org/fr/usagers/politique-dutilisation/ 


\section{Recension}

Durrive, L. (2015). L'expérience des normes. Comprendre l'activité humaine avec la démarche ergologique. Toulouse: Octares éditions.

\section{Présentation}

Le nouvel ouvrage de Louis Durrive, professeur à la faculté des sciences de l'éducation de l'Université de Strasbourg, vise à faire découvrir l'activité humaine avec l'approche ergologique $^{1}$ et donc à contribuer à faire connaître et à diffuser cette approche. Cet ouvrage se centre en effet sur l'«expérience des normes» des individus et par conséquent sur la "renormalisation" dont ils font preuve en permanence en tant qu'êtres vivants. Il approfondit tout particulièrement l'analyse de cette expérience des normes, qui est indispensable pour produire des "agirs efficaces" dans le contexte particulier des activités de travail et de formation, des activités humaines pour lesquelles la question des agirs efficaces est primordiale. En interaction avec des référentiels, des tâches ou des modalités pédagogiques prescrites, des règles de métier, etc., les travailleurs et les apprenants sont en effet présentés comme étant en permanence en quête d'un tel agir à travers l'expérience qu'ils font de ces différentes sources de production de normes.

Concept majeur au sein de l'approche ergologique, la renormalisation apparaît comme un excellent outil pour comprendre que les individus au travail ou en situation de formation sont loin d'être déterminés intégralement par les normes sociales qui les précèdent et les environnent, et qu'ils «retravaillent» au contraire délibérément ces normes, en les jaugeant, en les mettant en débat sans cesse. Plus précisément, afin de trouver une manière efficace d'agir sur le plan professionnel ou formatif, ils acceptent et contestent en même temps pareilles normes. Ce faisant, l'ouvrage de Louis Durrive rappelle combien la renormalisation est utile elle-même aux normes sociales et plus largement à la vie en société, en donnant sens, mouvement, chair à ces normes. Certes, affirme l'auteur, les normes requièrent l'idée d'un certain respect, d'une certaine conformité. Mais, pour

\footnotetext{
Conçue sous l'impulsion du philosophe Yves Schwartz à partir des années 1980, cette approche, appelée aussi ergologie, n'est pas une nouvelle discipline dans le champ des sciences humaines, mais une démarche qui vise à mieux connaître les situations de travail et surtout à mieux intervenir sur celles-ci pour les transformer.
} 
exister, démontrer leur utilité et finalement perdurer, elles ont besoin en même temps d'être retravaillées singulièrement et localement par les individus. C'est d'ailleurs la condition pour qu'elles puissent redevenir pleinement opérationnelles.

Cette renormalisation implique, selon l'auteur, un certain "usage de soi" dans le cadre de l'activité, qui passe à la fois par un "usage de soi par les autres" et un "usage de soi par soì. Usage de soi par les autres, car on travaille ou on apprend toujours en partie selon le point de vue d'autres acteurs, à partir de leur regard, de leurs attentes, de leur propre intervention dans le cours de l'activité. Usage de soi par soi, car dans ces situations, on fait toujours preuve d'initiative face aux contraintes et aux limites que l'on rencontre, tout en les prenant en compte pour tenter d'agir avec efficacité.

Les deux facettes incontournables de la renormalisation sont par ailleurs identifiées. Le travail singulier de la norme passe d'abord par son "actualisation» par l'individu, qui se pose dès lors comme acteur. Celui-ci la réaménage en situation, par exemple en tenant compte de ce que l'on attend de lui ici et maintenant. II n'applique jamais mécaniquement la norme en question, mais au contraire la retravaille à chaque fois, au moins partiellement, chaque situation rencontrée étant unique. Par exemple, il tient compte tout à la fois de ce que font au même moment ses collègues de travail, de ce que lui demande l'emploi spécifique qu'il occupe en termes d'objectifs à atteindre ou de tâches à réaliser, de ce qu'exigent son métier et la culture professionnelle qui lui est propre, etc.

La renormalisation induit ensuite une "personnalisation" de la norme. L'actualisation suppose en effet que l'individu arbitre entre plusieurs manières d'agir envisageables. II réévalue ainsi à partir d'un certain point de vue la valeur de la norme à laquelle il est confronté et opte finalement pour une certaine manière d'agir parmi plusieurs possibilités qui s'offraient a priori à lui. Il le fait en particulier en fonction de sa sensibilité et de ce qui "vaut" pour lui. Il se pose dès lors comme auteur de sa propre vie ou destinée. Il transforme la norme antécédente en sa propre norme. Concrètement, en situation professionnelle ou de formation, cela signifie qu'il en vient à adopter un style particulier, à privilégier certaines valeurs ou priorités personnelles, etc.

L'auteur insiste sur le fait que ces processus ne s'opèrent pas seulement sur le plan individuel, par la voie de l'affirmation et de la défense d'un point de vue particulier. Ils s'effectuent aussi sur le plan collectif, toute activité humaine étant forcément sociale, menée de concert ou en opposition avec autrui. Puisqu'il est question ici d'activités de travail et de formation exercées en entreprise ou dans les centres de formation, la recherche constante d'un agir efficace comporte de fait une large dimension collective. Afin de favoriser cette recherche, Louis Durrive propose d'ailleurs un nouveau modèle de management au sein des organisations, appelé «ergo-management». Celui-ci vise 
à introduire une rupture avec la conception classique du management dans laquelle tout est censé être réglé par du prescrit, et à promouvoir une conception beaucoup plus ergologique, c'est-à-dire davantage ouverte aux débats de normes et plus centrée sur la notion d'action collective.

Toutes ces analyses sont développées au sein d'un ouvrage structuré en quatre parties très complémentaires. La première partie porte sur ce que signifie "faire l'expérience des normes". La deuxième partie aborde la question de l'«usage de soi». La troisième partie analyse les modalités pour "être compétent", c'est-à-dire pour produire un agir efficace. La quatrième et dernière partie propose des pistes pour favoriser les débats de normes personnelles et collectives, et aider les acteurs à approfondir leur point de vue "en problématisant» (ergo-management, conditions pour produire des savoirs à partir de l'activité....).

\section{Point de vue ${ }^{2}$}

Le grand mérite de l'ouvrage de Louis Durrive est sans aucun doute d'approfondir le concept de renormalisation, au cœur de l'approche ergologique en lien avec la production des agirs efficaces. L'auteur sait de quoi il parle. C'est un chercheur porteur de cette approche depuis plus de 20 ans, mais aussi un ancien professionnel de l'éducation et de la formation spécialisé dans le domaine de la transition école-entreprise, et ayant été confronté à ce titre à des problématiques et à des interrogations très concrètes justifiant la convocation de pareille approche.

Dans cette perspective d'approfondissement, l'auteur retrace utilement la généalogie et la dynamique des normes. Celles-ci ont directement partie liée aux manières de faire, d'agir, de vivre ensemble. Elles remontent de fait aux origines de l'humanité et naissent fondamentalement du phénomène de "double anticipation" qui est propre à l'être humain. Celui-ci a la capacité de s'extraire du présent de l'action. Il pense à l'avance, organise, fait un programme, planifie et dit finalement a priori ce qui doit être (première anticipation). En même temps, il ne peut pas éluder le présent et se trouve confronté dans la réalité à des contraintes qui vont inévitablement déborder ce qu'il a déjà anticipé et l'amener à reprendre l'initiative (seconde anticipation). On peut se demander toutefois si cette dynamique des normes agit toujours au même rythme et sous la même forme, par simple transformation partielle des normes antécédentes sous l'effet de cette double anticipation. En particulier, qu'est-ce qui se passe quand certaines normes deviennent soudainement

$\overline{2}$ Ce point de vue a la particularité de ne pas seulement procéder de notre lecture personnelle de l'ouvrage de Durrive. Il se nourrit aussi des discussions et des échanges tenus dans le cadre d'une séance du Séminaire Analyse du Travail du Centre d'études et de recherches sur les qualifications, qui a eu lieu le 13 octobre 2015 dans les locaux de ce dernier à Marseille. Cette séance s'était notamment appuyée sur une intervention de l'inspecteur général du ministère de l'éducation nationale, Jean-Michel Paguet, en qualité de discutant. 
caduques lorsque, par exemple, l'environnement technologique, en situation de travail, connaît une réelle rupture, à l'image de ce qui semble se produire actuellement avec la révolution numérique? Il y aurait probablement lieu de continuer ainsi à sophistiquer le modèle d'analyse en mettant la focale sur les contextes de travail ou de formation, où la dynamique des normes doit être à la fois rapide et intense.

Louis Durrive en vient aussi à reconstruire habilement le concept de "compétence", alors que celui-ci semblait passé de mode après avoir été autant usité, sinon galvaudé. Il contribue notamment à en définir mieux les contenus et les contours. II entend à juste titre maintenir la compétence comme un discours normatif, cette fois ne portant plus sur les supposées qualités indéniables et immuables de l'individu concerné, comme le font les modèles et les pratiques de gestion par les compétences les plus répandues, mais sur I'usage que cet individu fait de lui-même en situation. En reprenant les travaux d'Yves Schwartz, il adopte une approche de la compétence qui comprend plusieurs "ingrédients" et s'appuie sur l'idée que l'acte et la personne sont en permanence en devenir. Certains ingrédients décrivent l'acte (par ex.: l'ensemble des normes antécédentes, telles que les savoirs, les savoirs d'organisation, les savoirs de métier à mobiliser et à combiner), les autres, la personne (par ex.: son histoire, ses ressources; la dimension sociale du travail et les synergies nouées avec les autres). Cette conception de la compétence permet toutefois de la distinguer de la notion de qualification. Cette dernière apparaît très abstraite, généraliste, extérieure aux individus, anonyme, alors que la compétence joue directement sur la situation dans laquelle intervient et s'engage à titre personnel l'individu.

Partant, la manière par laquelle l'auteur conçoit l'évaluation des compétences, à travers notamment l'usage des référentiels (de diplôme ou d'entreprise), apparaît tout à fait pertinente et convaincante. En effet, il y aurait lieu, d'après lui, d'évaluer les compétences selon plusieurs points de vue à croiser. Cela signifie qu'il faudrait pouvoir compter effectivement sur des référentiels, c'est-à-dire sur des "normes antécédentes" (une liste d'actes référencés et reconnus comme significatifs de la compétence professionnelle). Mais en même temps, il faudrait pouvoir aussi redonner toute sa place à l'acteur, au travers d'un débat de points de vue qui va permettre de considérer concrètement son usage de soi en situation: par exemple, voir comment il a profité des synergies collectives, suscité lui-même des synergies, ou su tenir compte des autres, notamment en s'effaçant là où il n'était pas lui-même compétent. De ce fait, les référentiels devraient toujours être utilisés dans le cadre d'un véritable débat, au cours duquel l'apprenant ou le salarié a réellement la possibilité de défendre son point de vue.

Par ailleurs, un lien subtil est établi entre savoirs et compétences, par la dialectique "désadhérence-adhérence". D'une part, les savoirs constitués (savoirs académiques, savoirs d'organisation...) sont hérités ou transmis de manière formelle. Ils renvoient à 
l'image de la désadhérence par rapport à la situation. D'autre part, les compétences ou les savoirs d'expérience sont ceux que l'on développe en situation pour faire face aux enjeux du présent et prendre des initiatives. Ils réfèrent quant à eux à l'image de l'adhérence. Cette analyse invite toutefois le lecteur à s'interroger sur le rôle de la formation professionnelle dans ce cadre. Est-ce, avant tout, de faire vivre aux personnes des situations de travail et de leur faire prendre conscience des savoirs d'expérience qu'elles en tirent, en décentrant ces savoirs d'expérience par rapport à ces situations? Ou bien plutôt transmettre des savoirs académiques, en apprenant aux personnes à les utiliser en situation professionnelle?

Enfin, on peut souligner l'intérêt que représente l'ergo-management, qui conduit à regarder autrement le travail, non pas de façon monotone et statique en se limitant à ce qui est a priori à faire, mais de manière plus vivante, tel qu'il se fait réellement. On assiste du reste depuis quelques années à un développement des expériences visant à mettre en place ce mode de management au sein de divers milieux de travail. En outre, l'esprit de ce dernier irrigue de façon informelle la pratique quotidienne de nombreux managers, sensibles à l'activité de travail telle qu'elle se fait, en complément de sa conception et de son organisation préalables. Cependant, on est en droit de se demander quelle est aujourd'hui l'importance de la diffusion effective de l'ergo-management et quel est son caractère opérationnel. Pour tenter de dépasser le stade enviable mais encore insuffisant de simple utopie réaliste, ne convient-il pas, au-delà des expérimentations localisées ici et là et des actions de sensibilisation et de formation des cadres à ce style de management, que les organisations inventent et développent aujourd'hui des fonctionnements collectifs globaux allant en ce sens? Ces fonctionnements collectifs favoriseraient autant que possible la mise en débat permanente des différentes normes à l'œuvre (travail prescrit, règles de métier, normes du collectif d'appartenance...) par tout un chacun dans son activité de travail. Pour nous, il en va d'ailleurs plus que jamais de la santé et du développement professionnel des travailleurs, mais aussi de l'efficacité et de la performance durables des organisations sur le plan économique.

\section{Samira Mahlaoui}

Docteure en sciences de l'éducation Ingénieure de recherches en analyse du travail et responsable de l'animation nationale du réseau des centres associés Centre d'études et de recherches sur les qualifications (Céreq) Marseille (France) 